\title{
Constraining Extended Theories of Gravity using Solar System Tests
}

\author{
Gianluca ALLEMANDI \\ Dipartimento di Matematica, \\ Università di Torino, Via C. Alberto 10, 10123 Torino \\ and INFN, Sezione di Torino \\ Matteo Luca RUGGIERO \\ Dipartimento di Fisica, \\ Politecnico di Torino, Corso Duca degli Abruzzi 24, 10129 Torino \\ and INFN, Sezione di Torino
}

(Dated: October 2, 2018)

\begin{abstract}
Solar System tests give nowadays constraints on the estimated value of the cosmological constant, which can be accurately derived from different experiments regarding gravitational redshift, light deflection, gravitational time-delay and geodesic precession. Assuming that each reasonable theory of gravitation should satisfy Solar System tests, we use this limits on the estimated value of the cosmological constant to constrain extended theories of Gravity, which are nowadays studied as possible theories for cosmological models and provide viable solutions to the cosmological constant problem and the explanation of the present acceleration of the Universe. We obtain that the estimated values, from Solar System tests, for the parameters appearing in the extended theories of Gravity are orders of magnitude bigger than the values obtained in the framework of cosmologically relevant theories.
\end{abstract}

PACS numbers: 98.80.Jk, 04.50.+h, 04.20.-q

\section{INTRODUCTION}

The fact that the Universe is now undergoing a phase of accelerated expansion is evidenced by many recent observational tests, such as the light curves of the type Ia supernovae and the cosmic microwave background (CMB) experiments [1, 2, 3, 4, 5, 6]. The present acceleration of the Universe can be explained, within General Relativity (GR), by claiming the existence of the dark energy, a cosmic fluid having exotic properties, since it is required to have equation of state such that $p<-\rho$, i.e. it has negative pressure smaller than its energy density.

Alternatively, it has been suggested that cosmic speed-up can be explained by generalizing GR: introducing in the gravitational action terms non linear in the scalar curvature $R$ (see [7, [8] and references therein). These theories are the so called $f(R)$ theories of gravity (or "extended theories of gravity"), where the gravitational Lagrangian depends on an arbitrary analytic function $f$ of the scalar curvature $R$. It was also shown recently that $f(R)$ gravity is a cosmologically viable theory because it may contain matter dominated and radiation phase before acceleration [9].

In spite of the apparent difficulties in agreeing with the recent cosmological observations, GR is in excellent agreement with the Solar System experiments: hence, every theory that aims at explaining the accelerated expansion of the Universe, should reproduce GR at the Solar System scale. Then, there is the need of check-

\footnotetext{
*Electronic address: allemandi@dm.unito.it
}

${ }^{\dagger}$ Electronic address: matteo.ruggiero@polito.it ing $f(R)$ predictions with Solar System tests or imposing constraints that derive from the experiments at Solar System scale. Actually, the debate is still open and, since $f(R)$ theories can be studied both in the metric formalism 10, 11, 12, 13 and in the Palatini formalism $14,15,16,17,18,19,20,21$, the problem can be faced within both approaches (see [22] and references therein).

Actually, we must say that there is not a common agreement on the dynamics of $f(R)$ theories in presence of matter, i.e. inside the sources of the gravitational field, both in the metric and in the Palatini formalisms [23, 24].

Some recent papers state that $f(R)$ theories do not match Solar System Tests [25, 26, 27]. Indeed, these analyses are carried out in the metric formalism and lead to the same results obtained some years ago by Chiba [23]. For a different viewpoint, always in the metric formalism, which leads to an agreement with Solar System Tests, see [28, 29]. The difficulties arising in the metric formalism with the Solar System experiments, which ultimately depend on the matching between the solutions inside and outside the matter distribution, are not present in the Palatini formalism, as it has been recently showed [30]: in fact, in this case the space-time inside a star does not affect the space-time outside it, (i.e. the vacuum solution), contrary to what happens in the metric formalism. Also, it is useful to remember that the solutions of the Palatini field equations are a subset on solutions of the metric field equations: thus Palatini $f(R)$ theories differ strongly from metric $f(R)$ theories theory [31].

In a previous work, we found an exact solution of the field equations in vacuum, in the Palatini approach and examined the significance of Post-Newtonian parameters thus arising [32]. This solution corresponds to the Schwarzschild-de Sitter metric, already studied in detail 
in [33], and the modifications to GR are related to the solutions of a scalar valued equation, that we called the structural equation. Owing to this fact, we showed that the GR limit (i.e. the Schwarzschild solution) and the Newtonian limit are recovered in the Solar System scale, thus suggesting the reliability of $f(R)$ at this scale. On the other hand, the question arises of evaluating the constraints, coming to Solar System experiments, on the $f(R)$ theories and, in particular, on the allowed analytical functions $f$ of the curvature scalar. Thanks to the exact Schwarzschild-de Sitter solution, we suggest here that this task can be, in principle, accomplished, according to a procedure that has been introduced in [34]. In fact, recent papers by [35, 36, 37] regarding the constraints on the Schwarzschild-de Sitter metric coming for the Solar System experiments, provide a method to give the best estimate for the parameter $k$ which, in our approach, is a measure of the non-linearity of the theory. What we ultimately aim at is obtaining constraints on the parameters appearing in the $f(R)$ functions, that is to say on the allowed forms of $f(R)$. As a by product, since our approach lends itself to set limits on the allowable values of the scalar curvature $R$, we may infer corresponding bounds on the cosmological density of (visible) matter.

\section{CONSTRAINTS ON $f(R)$ FROM SOLAR SYSTEM EXPERIMENTS}

The field equations of $f(R)$ theories, in the Palatini formalism, which have the explicit form:

$$
\begin{aligned}
f^{\prime}(R) R_{(\mu \nu)}(\Gamma)-\frac{1}{2} f(R) g_{\mu \nu} & =\kappa T_{\mu \nu}^{m a t} \\
\nabla_{\alpha}^{\Gamma}\left[\sqrt{g} f^{\prime}(R) g^{\mu \nu}\right) & =0
\end{aligned}
$$

admit the spherically symmetric vacuum exact solution:

$$
\begin{aligned}
d s^{2}= & -\left(1-\frac{2 M}{r}+\frac{k r^{2}}{3}\right) d t^{2}+\frac{d r^{2}}{\left(1-\frac{2 M}{r}+\frac{k r^{2}}{3}\right)} \\
& +r^{2} d \vartheta^{2}+r^{2} \sin ^{2} \vartheta d \varphi^{2}
\end{aligned}
$$

which is commonly referred to as Schwarzschild-de Sitter metric [41]: we refer to [32] for details on the derivation of this solution. The parameters appearing in (31) are the mass $M$ of the spherically symmetric source of the gravitational field and $k$, which is related to the solutions $R=c_{i}$ of the structural equation

$$
f^{\prime}(R) R-2 f(R)=0 .
$$

controlling the solutions of equation (11). Namely, it is $k=c_{i} / 4=R / 4$. We point out that (3) is a constant curvature solution. Indeed, we may say that $k$ is a measure of the non-linearity of the theory (if $f(R)=R$, eq. (44) has the solution $R=0 \rightarrow k=0$ ). It is evident, at least from a theoretical viewpoint, that the $f(R)$ contribution to the gravitational potential should be small enough not to contradict the known tests of gravity. In the cases of small values of $R$ (which surely occur at Solar System scale) the Einsteinian limit (i.e. the Schwarzschild solution) and the Newtonian limit are recovered, as it is manifest from (3).

On the other hand, inspection of the Schwarzschild-de Sitter metric, suggests that, when $M=0$ the (constant curvature) solutions are not, in general, Minkowski space-time, but they should be de Sitter or anti de Sitter space-time: as a matter of facts, Minkowski space-time is solution of the field equations only if $R=0$ (see [32]).

In GR, the Schwarzschild-de Sitter solution corresponds to a spherically symmetric solution of Einstein equations, with a "cosmological" term $\Lambda g_{\mu \nu}, \Lambda$ being the cosmological constant, which might be, in turn, one of the candidates for explaining the accelerated expansion of the Universe, even though the nature of the cosmological constant is still under debate [38]. In practice, it is $\Lambda=-k$ in our notation. A recent paper by Kagramanova et al. 35] calculates the classical gravitational effects in the Solar System on the basis of the Schwarzschild-de Sitter metric. On studying the gravitational redshift, the deflection of light, the gravitational time-delay and the geodetic precession, constraints are deduced on the magnitude of the cosmological constant. Besides the deflection of light, which is not influenced by $\Lambda$, the other tests give estimates on $\Lambda$ that are much greater than those estimated from observational cosmology. Indeed, these estimates range from $|\Lambda| \leq 10^{-24} \mathrm{~m}^{-2}$ to $|\Lambda| \leq 10^{-27} \mathrm{~m}^{-2}$ while, in cosmology, the current value of the cosmological constant is estimated to be $\Lambda_{0} \simeq 10^{-52} m^{-2}$ [38].

Using a different approach, Jetzer and Sereno [36] suggest that the best constraint $\Lambda_{0} \simeq 10^{-42} \mathrm{~m}^{-2}$ comes from the extra precession on the basis of Earth and Mars observations. A similar result was also obtained by Iorio [37.

Armed with this results, we may reverse the argument, and obtain, from these constraints on $|\Lambda|=|k|$, limits on the $f(R)$ functions used in cosmology to explain cosmic speed-up. This is a further test to obtain constraints on $f(R)$ gravitational theories and to test their viability with well accepted experimental tests for Gravity.

As we have seen, the $k$ parameter is simply related to the solutions of the structural equation

$$
f^{\prime}(R) R-2 f(R)=0,
$$

namely it is $k=c_{i} / 4$, where $R=c_{i}$ are the solutions of (51). As a consequence, the bounds on the $k$ parameter, in principle, enable us to constrain the functions $f(R)$. We may proceed as follows. In general, the functions $f$, beyond the scalar curvature $R$, depend on a set of $N$ constant parameters $\alpha_{j}, j=1 . . N$, so that we may write $f=f\left(R, \alpha_{1}, . ., \alpha_{N}\right)$, and, on solving (5), we obtain

$$
R=\mathcal{F}\left(\alpha_{1}, . ., \alpha_{N}\right) .
$$

Consequently, what we ultimately obtain is a limit on the allowed values of the combination $\mathcal{F}$ of these parameters. 
For instance, let us considered the Lagrangian introduced in [7], which mimics cosmic acceleration without need for dark energy:

$$
f(R)=R-\frac{\mu^{4}}{R} .
$$

We point out that the Lagrangian (7) was found to be instable 10, 11, 39]. For discussion on this issue, we refer to the recent paper by Faraoni [40]. Nonetheless, we consider the Lagrangian (7) as a first example in order to illustrate our approach.

The Lagrangian (7) depends on the parameter $\mu$ only, so that eq. (6) becomes

$$
|R|=\sqrt{3} \mu^{2}
$$

and, consequently, we may set a limit on the parameter $\mu$. If we take the value $|\Lambda| \leq \times 10^{-42} m^{-2}$ from [36], we get

$$
\mu \leq 10^{-21} m^{-1}
$$

or, since $\mu$ is a parameter whose physical units are those of a mass,

$$
\mu \leq 10^{-28} \mathrm{eV}
$$

We can alternatively use the best estimate $|\Lambda| \leq$ $10^{-27} \mathrm{~m}^{-2}$ given in 35] and we correspondingly get

$$
\mu \leq 10^{-20} \mathrm{eV}
$$

We notice that both values (10]11) are remarkably greater than estimate $\mu \simeq 10^{-33} \mathrm{eV}$ [7], needed for $f(R)$ gravity to explain the acceleration of the Universe without requiring dark matter.

A most significant example is given by polynomiallike functions $f(R)$; it can be showed that the structural equation can be solved analytically. This is the case already examined in [15]:

$$
f(R)=\alpha R+\frac{\beta}{m-2} R^{m}(m \neq 1,2),
$$

from which we obtain, on solving the structural equation:

$$
|\mathcal{F}(\alpha, \beta, m)|=\left|\frac{\alpha}{\beta} \frac{1}{1-m}\right|,
$$

This implies that the same bounds can be derived on the function $\mathcal{F}(\alpha, \beta, m)$ :

$$
|\mathcal{F}(\alpha, \beta, m)| \leq 10^{-42} m^{-2}
$$

or

$$
|\mathcal{F}(\alpha, \beta, m)| \leq 10^{-27} m^{-2},
$$

according to [36] or 35] respectively. This example is relevant from a cosmological viewpoint: the present form of $f(R)$ reproduces an inflationary behavior of the model, in the case $m>0$ and, on the contrary, the case $m<0$ is important to construct and study accelerated models of the present Universe as previously remarked.

We can also consider the Lagrangian [14, 15]

$$
f(R)=R-\frac{6 \alpha}{\sinh R / \alpha},
$$

in the limit of small $R$, such that the structural equation gives

$$
|R|=3 \alpha
$$

Consequently, we may set limits on the parameter $\alpha$, in particular

$$
\alpha \leq 10^{-42} m^{-2}
$$

and

$$
\alpha \leq 10^{-27} m^{-2}
$$

following to [36], or 35], respectively. According to [15], we must take $\alpha \simeq 10^{-52} m^{-2}$ to match observations of the cosmological acceleration, and, once again, the cosmologically relevant values are much smaller than those obtained from Solar System experiments.

What we have seen suggests that the constraints deriving from Solar System tests are surely coherent with cosmological theories in the framework of extended theories of Gravity, which are able to explain the present acceleration of the Universe. The feeling is that data coming from one context cannot be used to infer anything on the other context, since they have very different orders of magnitude.

The dichotomy between Solar System experiments and cosmological observations can be seen from a different viewpoint. As we have said, $f(R)$ theories of gravity are expedient to explain cosmic speed-up, thanks to a geometrical approach, without requiring the existence of dark energy or other exotic fluids. This can be accomplished by studying the Friedman-Robertson-Walker (FRW) cosmology in $f(R)$ gravity [15]. In this case, the structural equation (4) reads

$$
f^{\prime}(R) R-2 f(R)=\kappa \tau,
$$

where $\tau=\operatorname{tr} T=g^{\mu \nu} T_{\mu \nu}^{\text {mat }}, T_{\mu \nu}^{\text {mat }}$ being the stress-energy tensor of the cosmological fluid. Re-phrasing the approach outlined above, the solution of eq. (20) can be written in the form

$$
R=\mathcal{G}\left(\alpha_{1}, . ., \alpha_{N}, \tau\right)
$$

In other words, what we can get is a limit on the allowed values of the combination $\mathcal{G}$ of the $\alpha_{j}$ parameters and the trace $\tau$ of the stress-energy tensor of the cosmological fluid.

For instance, from the Lagrangian (7), we obtain

$$
R=\mathcal{G}(\mu, \tau)=\frac{-\tau \pm \sqrt{\tau^{2}+12 \mu^{4}}}{2} .
$$


If we consider the case of dust-dominated Universe, $\tau=$ $-\rho$ : as a consequence, (22) allows to get information on the allowed values of the combination of the matter density $\rho$ and the parameter $\mu$. Actually, since the parameter $\mu$ is required to be very small in order to match the cosmic speed-up [14], the relation (22) sets limits on the values of the (visible) matter density $\rho$, which, thanks to the data reported by [36] or 35], are easily seen to be much different from those deriving from cosmological observations.

\section{DISCUSSIONS AND CONCLUSIONS}

The recent estimates of $k$ (or $\Lambda$ ) from Solar System experiments suggest some comments on the reliability of extended theories of gravity.

First of all, up today, no observation within the Solar System is able to reveal effects due to the non linearity of $f(R)$ (or, rephrased in GR language, due to the cosmological constant). Strictly speaking, this statement is the outcome of our analysis that has been carried in the Palatini formalism of $f(R)$ gravity; however similar conclusions seem to arise also in the metric approach, as recent papers suggest (see [28, 29] and references therein).

Second, conversely, present observations at the Solar System scale are hardly fit to constrain the forms of the $f(R)$ functions used in cosmology, setting bounds on the parameters appearing in them, since the Solar System estimates of these parameters are much greater than the cosmologically required values. This is confirmed also by estimating the density of visible matter, which turns out to be several orders of magnitude different from the one derived thanks to cosmological observations.

Of course, these tests are done on a particular solution of the $f(R)$ equations, namely a spherically symmetric vacuum solution, corresponding to constant scalar curvature. Even if relaxing the spherical symmetry does not seem to produce relevant changes, different conclusions might arise considering the solutions inside a matter distribution, such as a galaxy, thus enlarging the scale under consideration.

The underlying feeling is that non-linearities appearing in $f(R)$ become important on scales much larger than the Solar System, for sure on the cosmological scale and, hence, data coming from one context cannot be used to infer anything on the other context: in fact, they belong to a different realm of application of the same theory, if one trusts in $f(R)$, or to different approximations of a more general theory, if one believes that GR at the Solar System scale and $f(R)$ in cosmology are just good approximations of something else, still undefined.

\section{ACKNOWLEDGMENTS}

M.L.R acknowledges financial support from the Italian Ministry of University and Research (MIUR) under the national program "Cofin 2005" - La pulsar doppia e oltre: verso una nuova era della ricerca sulle pulsar.
[1] Riess A. G. et al. AJ 116, 1009 (1998), astro-ph/9805201

[2] Perlmutter S. et al., ApJ 517, 565 (1999), astro-ph/9812133

[3] Tonry J.L. et al., ApJ 594 1, (2003), astro-ph/9305008

[4] Bennet, C.L. et al, ApJ Suppl. 148, 1 (2003), astro-ph/0302207

[5] Netterfield et al., ApJ 571, 604 (2002), astro-ph/0104460

[6] Halverson N.W. et al., ApJ 568, 38 (2002), astro-ph/0104489

[7] Carroll S.M., Duvvuri V., Trodden M., Turner M.S., Phys. Rev. D70, 043528 (2004), astro-ph/0306438

[8] Nojiri S., Odintsov S.D., Lectures given at 42nd Karpacz Winter School of Theoretical Physics, hep-th/0601213 (2006)

[9] Nojiri S., Odintsov S.D., Phys.Rev. D74, 086005 (2006), hep-th/0608008 Nojiri S., Odintsov S.D., paper prepared for Special Issue IRGAC 2006, Barcelona, hep-th/0610164 Capozziello S., Nojiri S., Odintsov S.D., Troisi A., Phys.Lett. B639, 135 (2006), astro-ph/0604431

[10] Nojiri S., Odintsov S.D., Phys. Rev. D68, 123512 (2003), hep-th/0307288

[11] Nojiri S., Odintsov S.D., Gen. Rel. Grav. 36, 1765 (2004), hep-th/0308176

[12] Nojiri S., Odintsov S.D., Mod. Phys. Lett.A 19, 627 (2004), hep-th/0310045
[13] Olmo G. J., Phys. Rev. D72, 083505 (2005)

[14] Vollick D.N., Phys. Rev. D68, 063510 (2003), astro-ph/0306630

[15] Allemandi G., Borowiec A., Francaviglia M, Phys.Rev. D70, 043524 (2004), hep-th/0403264

[16] Olmo G.J., Phys. Rev. Lett. 95 (2005) 261102, gr-qc/0505101

[17] Olmo G.J., Komp W., gr-qc/0403092

[18] Meng X.H., Wang P., Class. Quant. Grav. 20, 4949 (2003), astro-ph/0307354

[19] Meng X.H., Wang P., Class. Quant. Grav. 21, 951 (2004), astro-ph/0308031

[20] Kremer G.M., Alves D.S.M., Phys.Rev. D70, 023503 (2004), gr-qc/0404082

[21] Allemandi G., Borowiec A., Francaviglia M., Odintsov S.D., Phys.Rev. D72, 063505 (2005), gr-qc/0504057

[22] Faraoni V., Phys.Rev. D74, 023529 (2006), gr-qc/0607016

[23] Chiba T., Phys.Lett. B 575, 1 (2003), astro-ph/0307338

[24] Flanagan É. É. , Phys.Rev.Lett. 92, 071101 (2004), astro-ph/0308111

[25] Erickcek A.L., Smith T. L. , Kamionkowski M., Phys.Rev. D74 121501 (2006), astro-ph/0610483

[26] Jin X., Liu D., Li Z., astro-ph/0610854

[27] Chiba T., Smith T. L., Erickcek A.L., astro-ph/0611867 
[28] Capozziello S., Stabile A., Troisi A., Mod. Phys. Lett. A21, 2291 (2006)

[29] Zhang P., astro-ph/0701662

[30] Kainulainen K., Reijonen V., Sunhede D., gr-qc/0611132

[31] Magnano G., in General relativity and gravitational physics: Proceedings, Eds. Carfora M., Cavaglià M., Francaviglia M., Frè P., Pizzella G., Reina C., Treves A., World Scientific, Singapore (1996), gr-qc/9511027

[32] Allemandi G., Francaviglia M., Ruggiero M.L., Tartaglia A., Gen. Rel. Grav. 37, 1891 (2005), gr-qc/0506123

[33] Brevik I., Nojiri S., Odintsov S.D. and Vanzo L., Phys. Rev. D70, 043520 (2004), hep-th 0401073 Cognola G., Elizalde E., Nojiri S., Odintsov S.D. and Zerbini S., JCAP 0502 (2005), hep-th/0501096.

[34] Ruggiero M.L., Iorio L., JCAP, 0701010 (2007), gr-qc/0607093

[35] Kagramanova V., Kunz J., Lämmerzahl C., Phys.Lett. B 634, 465 (2006), gr-qc/0602002

[36] Jetzer P., Sereno M., Phys.Rev. D73, 044015 (2006), astro-ph/0601612

[37] Iorio L., JCAP 0605, 002 (2006), gr-qc/0602095

[38] Peebles P.J.E., Ratra B., Rev. Mod. Phys.75, 559 (2003), astro-ph/0207347

[39] Dolgov A.D., Kawasaki M., Phys. Lett. B 573, 1 (2003), gr-qc/0307285

[40] Faraoni V., Phys.Rev. D74, 104017 (2006) , astro-ph/0610734

[41] Our results hold if the Lagrangian is not in the form $f(R)=R^{n}$, with $n \geq 2, n \in \mathbb{N}$. 